\title{
SIMULTANEOUS OPTIMIZATION OF HYBRID FIBER AMPLIFIERS AND DISPERSION MAPS
}

\author{
Vitorrio Curri ${ }^{1,2}$ and Stefan Tenenbaum ${ }^{1}$ \\ ${ }^{1}$ Dipartimento di Eletronica, Politecnico di Torino,Torino, Italy, optcom@polito.it \\ ${ }^{2}$ ATS Srl, Torino, Italy, info@alps-telsoft.com
}

\begin{abstract}
Multi-span optical systems using Hybrid Fiber Amplifiers are optimized for the lowest $B E R$ under dispersion management for a $2000 \mathrm{Km}$ link operating at 42.7 $\mathrm{Gbit} / \mathrm{s}$. The best span configuration including optimum Raman/Erbium gain balance and ideal dispersion compensation degree is found. It is shown that hybrid amplifier configurations behave better than EDFA-only and Raman-only systems, accumulating less noise and nonlinearities.
\end{abstract}

\section{INTRODUCTION}

Hybrid Raman/Erbium-Doped Fiber amplifiers (HFA) are an excellent choice for in-line optical amplification in multi-span links thanks to the improvement of system Optical Signal-to-Noise Ratio $(O S N R)$ and broader bandwidth with respect to systems based on pure Erbium-Doped Fiber Amplifiers (EDFA). Furthermore, dispersion compensation and Raman gain can be integrated in a single unit [1-3].

In previous theoretical developments [4,5] the expression for the OSNR at the receiver was maximized varying the balance between Raman and EDFA gains, but there was no design of dispersion map since dispersion was assumed to be completely compensated at each span. In this work, we add to the previous analyses the simultaneous optimization of the dispersion map, i.e., the amount of in-line - length of the dispersion compensating fiber (DCF) inserted after each span - and total dispersion compensation - dispersion of the fiber grating $(\mathrm{FG})$ inserted before the receiver.

The optimization is performed using a semi-analytical approach, then results are verified a posteriori using the optical system simulator OptSim ${ }^{\circledR}{ }^{\circledR}$ [6] in order to verify the impairments induced by propagation effects (chromatic dispersion and nonlinearities). 


\section{SYSTEM CONFIGURATION}

The system configuration we considered is presented in Fig. 1. It is a multispan amplified optical link with $N_{S P A N}$ periods. Each period is composed of a transmission fiber span (whose length is $L_{S P A N}$ ) backward pumped in order to get Raman amplification (RA), a first EDFA, a dispersion compensating fiber (DCF) span whose length $L_{D C F}$ defines the degree of in-line dispersion compensation (and consequently the amount of in-line residual dispersion: $D_{\text {res, IL }}$ ), a gain flattening filter (GFF) of $4 \mathrm{~dB}$ loss and a second EDFA.

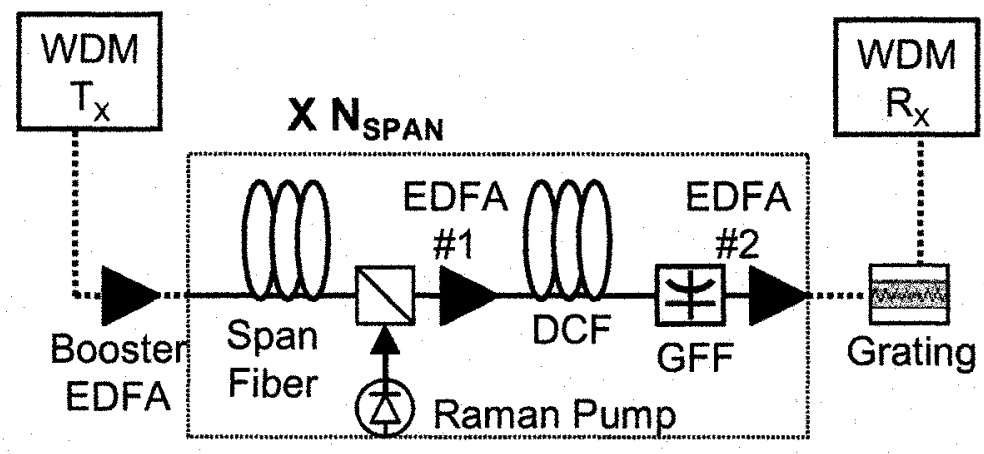

Figure 1. System setup used for the optimization.

After the $N_{S P A N}$ periods, we considered to insert an ideal fiber grating (FG) that contributes to the overall dispersion compensation and defines the total dispersion residue $\left(D_{\text {res,TOT }}\right)$. Contrary to the DCF, the FG does not introduce nonlinear effects. Here are the analytical expressions for $D_{r e s, I L}$ and $D_{r e s, T O T}$ :

$$
\begin{aligned}
D_{\text {res }, I L} & =D_{T F} \cdot L_{S P A N}+D_{D C F} \cdot L_{D C F} & & {[\mathrm{ps} / \mathrm{nm}] } \\
D_{\text {res }, T O T} & =N \cdot D_{\text {res, }, L}+D_{F G} & & {[\mathrm{ps} / \mathrm{nm}] }
\end{aligned}
$$

where $D_{T F}$ and $D_{D C F}$ are the dispersion coefficients, expressed in ps $/ \mathrm{nm} / \mathrm{Km}$, for the transmission fiber and for the DCF, respectively, and $D_{F G}$ is the amount of dispersion, expressed in ps/nm, introduced by the FG. The amplifiers completely recover the overall losses of each link period (losses of fibers + GFF loss).

Our analysis is applied to two system scenarios based on different transmission fibers: one based on SMF and the other based on non-zero dispersion shifted fiber (NZ-DSF). See Table 1 for transmission parameters of the considered fibers. We analyzed a link length of $2000 \mathrm{~km}$ subdivided in periods of $L_{S P A N}=50 \mathrm{~km}$ and $L_{S P A N}=80 \mathrm{~km}$. We assumed to use standard IM-DD NRZ modulation and a bit-rate $R_{B}=42.7 \mathrm{Gbit} / \mathrm{s}(40 \mathrm{Gbit} / \mathrm{s}+\mathrm{FEC}$ overhead).

The purpose of the work was to maximize the system OSNR (and consequently minimize the $B E R$ ) varying the balance between Raman and EDFA 
gains. With respect to previous works [4,5], we added to the analysis the simultaneous optimization of the amount of in-line and total dispersion compensation (DCF length and FG dispersion) exploring the possible dispersion maps with $D_{\text {res, } I L}$ varying from -30 to $30 \mathrm{ps} / \mathrm{nm}$ and $D_{\text {res,TOT }}$ from -100 to 100 $\mathrm{ps} / \mathrm{nm}$.

\section{THE ANALYSIS}

Using RAs, the impact of nonlinearities is stronger than in EDFA-only systems, because the span average signal power profile tends to be higher. Thus, the parameter $k_{N L}$ (nonlinear weight) that takes into account the accumulated nonlinear phase shift assumes the following expression [5]:

$$
\begin{aligned}
k_{N L} & =\int_{0}^{L_{L I N K}} \gamma(z) \cdot P(z) d z \\
& =N_{S P A N} \cdot P_{T X} \cdot\left[\gamma \cdot L_{e f f}+\gamma_{D C F} \cdot L_{e f f, D C F} \cdot G_{E I} \cdot G_{R A} \cdot \mathrm{e}^{-\alpha_{S} \cdot L_{S P A N}}\right]
\end{aligned}
$$

where $\gamma$ and $\gamma_{D C F}$ are the nonlinear coefficients, and $L_{e f f}$ and $L_{e f f, D C F}$ the effective lengths [7], of the transmission fiber and DCF, respectively. $G_{R A}$ is the Raman on-off gain [5] and $G_{E I}$ is the gain of the EDFA \#1. $P_{T X}$ is the transmitted power.

As defined in [4], the noise accumulated on the link is $N_{T O T}=N_{S P A N} \cdot N_{1}$, where $N_{1}$ is noise power generated after a single span. Being $P_{1}$ the power launched to obtain $k_{N L}=1$ with $N_{S P A N}=1$, we can express the launched power with the help of Eq. 2 as $P_{T X}=k_{N L} \cdot P_{1} / N_{S P A N}$. Using $k_{N L}$, the system OSNR measured over a noise bandwidth equal to $R_{B}$ after $N_{S P A N}$ spans is:

$$
O S N R=\frac{P_{T X}}{N_{S P A N} N_{I}}=\frac{k_{N L} P_{1}}{N_{S P A N}^{2} N_{I}}=\frac{k_{N L}}{N_{S P A N}^{2}} \operatorname{OSNR}_{1},
$$

with $O S N R_{1}=P_{1} / N_{1}$. The analytical expression for $O S N R_{1}$ defined in [4], is:

$$
O S N R_{1}=\frac{P_{1}}{h f R_{B}\left[\left(n_{e q, R A}+n_{s p, E I} \frac{G_{E_{1}}-1}{G_{R A} \cdot G_{E_{I}}}\right) e^{\alpha_{S} \cdot L_{S}}+n_{s p, E 2}\left(G_{E_{2}}-1\right)\right]},
$$

where $h f$ is the photon energy, $n_{s p, E 1}$ and $n_{s p, E 2}$ are the spontaneous emission factors [8] of the EDFAs and $n_{\text {eq,RA }}$ is the equivalent input noise factor [5] for the RA [5]. The optimal HFA setup (Raman/EDFA gain balancing) is the one that maximizes $O S N R_{1}$ for each $L_{S P A N}$ and for each $L_{D C F}$ (and consequently each value of $D_{r e s, I L}$ ). Therefore, the optimization is done on $O S N R_{1}$ [4] independently of $N_{S P A N}$, thus, for each $L_{S P A N}$ and $D_{\text {res, } I L}$, there exists an optimal HFA configuration (amount of gain of the RA, EDFA \#1 and EDFA \#2) that gives the optimal $O S N R_{1}$ (function of $L_{S P A N}$ and $D_{\text {res, }, L}$ ). 
After deriving the optimal HFA for each $L_{S P A N}$ and $D_{\text {res, }, L}$, we defined the $O S N R_{T A R G E T}$ as the value of $O S N R$ corresponding to the required system biterror rate $\left(B E R_{T A R G E T}\right)$, remembering that

$$
B E R_{T A R G E T} \simeq \frac{1}{2} \cdot \mathrm{e}^{-0.98 \cdot O S N R_{\text {TARGET }}}
$$

We considered $O S N R_{T A R G E T}=16 \mathrm{~dB}$ corresponding to a $B E R_{T A R G E T} \approx 10^{-16}$. Using Eq. 3, we were able to express the nonlinear weight as

$$
k_{N L}=N_{S P A N}^{2} \frac{O S N R_{\text {TARGET }}}{O S N R_{1}} .
$$

Defining the total link length $(2000 \mathrm{~km})$ and the span length (we considered $L_{S P A N}=50$ and $\left.L_{S P A N}=80 \mathrm{~km}\right), N_{S P A N}$ is consequently defined $\left(N_{S P A N}=40\right.$ and $N_{S P A N}=25$ ), and $O S N R_{\text {TARGET }}$ is fixed by imposing the target $B E R$. Therefore, in Eq. 6, $k_{N L}$ becomes dependent on the optimal $O S N R_{1}$ (that varies with $D_{\text {res,IL }}$ ), defining for each $D_{\text {res, } I L}$, the corresponding nonlinear weight.

At this point of the process, we had at our disposal, for each of the two considered span lengths, a set of optimal HFA configurations, each corresponding to a different amount of in-line dispersion compensation $\left(L_{D C F} \rightarrow D_{\text {res, }, L}\right)$. $D_{\text {res,TOT }}$ does not influence the HFA configuration because the FG does not introduce nonlinearities, and consequently to each optimal HFA may correspond the overall range of $D_{r e s, T O T}$.

In order to evaluate the optimal dispersion map $\left(D_{\text {res,IL }}, D_{\text {res,TOT }}\right)$, we needed to evaluate system performances for the set of possible system configurations. Therefore, we simulated the propagation for all the system setups using the optical system simulator OptSim ${ }^{\circledR}$ [6], deriving a set of values of the $Q$ factor $\left(Q=20 \cdot \log _{10}\left\{\operatorname{erfc}^{-1}(2 \cdot B E R)\right\} \mathrm{dB}\right)$. Each derived $Q$ value corresponds to a point of the explored plane $\left(D_{\text {res, } I L}, D_{\text {res, } T O T}\right)$, where points with different values of $D_{\text {res,IL }}$ refer to different optimal HFA configurations. Therefore, final results are surfaces of $Q$ factor in the $\left(D_{\text {res,IL }}, D_{\text {res,ToT }}\right)$ plane: one for each considered $L_{S P A N}(50$ or $80 \mathrm{~km}$ ) and type of transmission fiber (SMF or NZDSF). From the contour plots of these surfaces, the optimal dispersion maps can be deduced, i.e., the areas of the $\left(D_{\text {res, } I L}, D_{\text {res,TOT }}\right)$ plane where the $Q$ factor exceeds an established threshold. Besides being the optimal dispersion map areas, these correspond to optimal HFA configuration as well, yielding the simultaneous optimization of HFA and dispersion map.

\section{RESULTS}

Even assuming to use a FEC, we considered to operate with an $O S N R_{\text {TARGET }}$ as high as $16 \mathrm{~dB}$, corresponding to $B E R \approx 10^{-16}$, i.e., $Q \approx 18.4 \mathrm{~dB}$. Of course, these values refer to the absence of propagation impairments. Using such ref- 
Table 1. Transmission parameters of the considered fibers.

\begin{tabular}{ccccccc}
\hline & $\begin{array}{c}\alpha_{\mathrm{S}} \\
{[\mathrm{dB} / \mathrm{Km}]}\end{array}$ & $\begin{array}{c}\alpha_{\mathrm{P}} \\
{[\mathrm{dB} / \mathrm{Km}]}\end{array}$ & $\begin{array}{c}\mathrm{D} \\
{[\mathrm{ps} / \mathrm{nm}]}\end{array}$ & $\begin{array}{c}\mathrm{A}_{\mathrm{eff}} \\
{\left[\mu \mathrm{m}^{2}\right]}\end{array}$ & $\begin{array}{c}\gamma \\
{[1 / \mathrm{W} / \mathrm{Km}]}\end{array}$ & $\begin{array}{c}\mathrm{C}_{\mathrm{R}} \\
{[1 / \mathrm{W} / \mathrm{Km}]}\end{array}$ \\
\hline SMF & 0.2 & 0.3 & +16 & 80 & 1.27 & 0.4 \\
DCF & 0.5 & - & -100 & 25 & 4.1 & - \\
NZ-DSF & 0.2 & 0.3 & +5 & 55 & 1.85 & 0.6 \\
\hline
\end{tabular}

erence values, and plotting Eq. 6 in the following form:

$$
k_{N L}=\left(\frac{L_{T O T}}{L_{S P A N}}\right)^{2} \cdot \frac{O S N R_{T A R G E T}}{O S N R_{I}\left(L_{S P A N}, D_{r e s, I L}\right)},
$$

where $O S N R_{1}\left(L_{S P A N}, D_{\text {res, }, I L}\right)$ means $O S N R_{1}$ as function of $L_{S P A N}$ and $D_{\text {res, }, I L}$, we obtained the plots of Figs. 2 for the $k_{N L}$ level curves in the plane $\left(L_{S P A N}, D_{\text {res,IL }}\right)$. Fig. 2a and Fig. 2b refer to the use of SMF and NZ-DSF as transmission fiber, respectively. Each vertical line of the explored plane refers to a different HFA configuration. Highlighted horizontal lines refer to $L_{S P A N}=50$ and $80 \mathrm{~km}$, i.e., to the span lengths considered for the following analysis. The points crossing such values refer to the optimal HFA setups for each $D_{r e s, I L}$. It can be observed that increasing the amount of $D_{r e s, I L}, k_{N L}$ increases because of the presence of a longer DCF span.
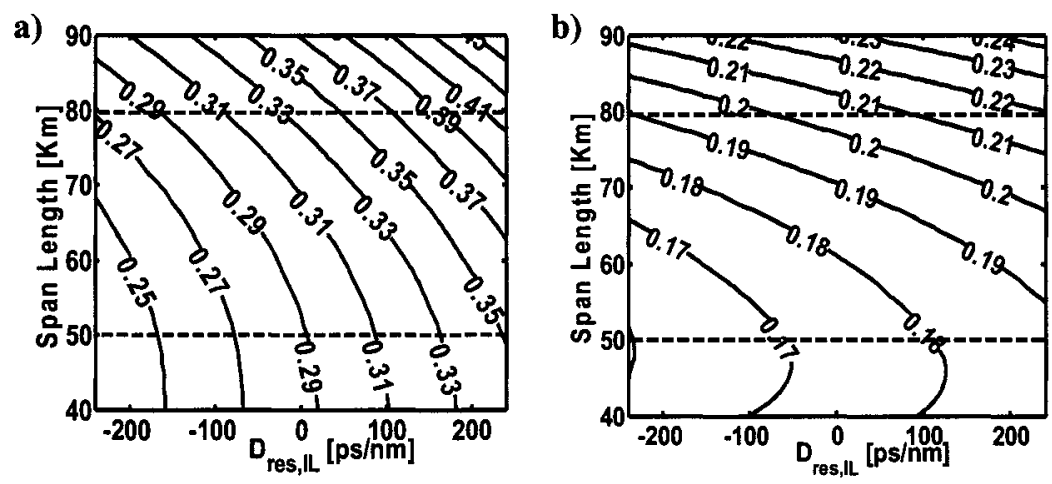

Figure 2. $k_{N L}$ in the $\left(D_{\text {res, }, L}, L_{S P A N}\right)$ plane for the case of using (a) SMF and (b) NZ-DSF.

After deriving the optimal HFA configurations for each considered scenario, we simulated the signal propagation for the corresponding system setup, varying $D_{\text {res,TOT }}$ beside varying $D_{\text {res, } I L}$. As previously described, variations of $D_{\text {res,TOT }}$ do not influence the optimal HFA configuration that depends only on 
$L_{S P A N}$ and $D_{\text {res, }, L}$. In order to define the optimal dispersion maps we swept $D_{\text {res, }, L}$ within $[-250 ;+250] \mathrm{ps} / \mathrm{nm}$ and $D_{\text {res,TOT }}$ within $[-100 ;+100] \mathrm{ps} / \mathrm{nm}$.

Resulting contour plots of $Q$ surfaces are presented in Fig. 3 for the following scenarios: $S M F$ and $L_{S P A N}=50 \mathrm{~km}$ (Fig. 3a), SMF and $L_{S P A N}=80 \mathrm{~km}$ (Fig. 3b), NZ-DSF and $L_{S P A N}=50 \mathrm{~km}$ (Fig. $3 \mathrm{c}$ ) and NZ-DSF and $L_{S P A N}=50$ $\mathrm{km}$ with pure Erbium amplification (Fig. 3d) as comparison.
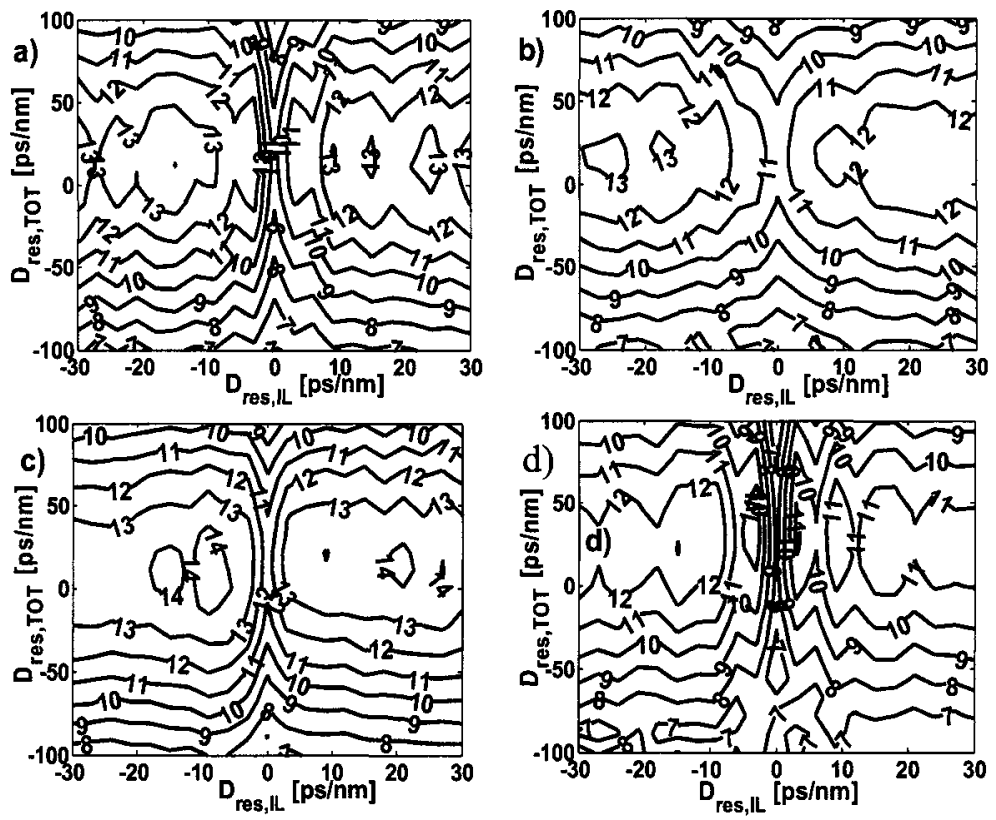

Figure 3. $Q$ level curves for (a) $S M F$ and $L_{S P A N}=50 \mathrm{~km}$, (b) $S M F$ and $L_{S P A N}=80 \mathrm{~km}$, (c) NZ-DSF and $L_{S P A N}=50 \mathrm{~km}$ and (d) SMF EDFA-only and $L_{S P A N}=50 \mathrm{~km}$, as comparison.

As can be observed on all graphs, the highest $Q$ value is found off the central point of the $\left(D_{\text {res, }, L}, D_{\text {res,TOT }}\right)$ plane, meaning that for the best transmission performance, dispersion should not be completely compensated (some in-line and total dispersion residue was left). This is a well known behavior due to the presence of nonlinearities - in particular, XPM - that is excited at the maximum if dispersion is totally compensated at each span [9].

In Fig. 3a it can be observed that using SMF as transmission fiber and $L_{S P A N}$ $=50 \mathrm{Km}$ a $Q_{M A X}=14 \mathrm{~dB}$ was obtained with $4.4 \mathrm{~dB}$ of $Q$ penalty due to propagation impairments, while for $L_{S P A N}=80 \mathrm{~km}$, the $Q_{M A X}$ decreases to $13 \mathrm{~dB}$ (Fig. $3 \mathrm{~b}$ ). Increasing $L_{S P A N}$ from $50 \mathrm{~km}$ to $80 \mathrm{Km}$, system performance decreases due to the enhancement of the nonlinear effects because:

- longer $L_{S P A N}$ implies longer DCF span that contributes to increase the $k_{N L}$ as we can see in Fig. 2a-b; 
- the longer is the span of transmission fiber the larger is the loss per span, therefore a larger amount of amplification is needed to completely recover the attenuation. It implies a higher noise power added to the signal, which consequently requires a higher amount of transmission power to obtain the target $O S N R$. Thus, the impact of nonlinearity is stronger and induces a larger penalty.

Using NZ-DSF and $L_{S P A N}=50 \mathrm{~km}$ (Fig. $\left.3 \mathrm{c}\right)$ the maximum $Q$ increases $\left(Q_{M A X}\right.$ $=14.5 \mathrm{~dB}$ ) with respect to the SMF case, because the larger nonlinear coefficient of the NZ-DSF - and consequent potential stronger nonlinear impact - is compensated by the need of a shorter span of DCF. Thus, the overall $k_{N L}$ is lower for the NZ-DSF + DCF system as it can be observed in Fig. 2c. Hence, a lower impairment of nonlinearities can be observed. A similar behavior characterizes the NZ-DSF with $L_{S P A N}=80 \mathrm{~km}$ scenario, whose results are not presented.

In order to understand the importance of Raman amplification we analyzed the same system scenarios using pure Erbium amplification. In Fig. 3d, we report results referred to $L_{S P A N}=50 \mathrm{Km}$ and use of SMF, i.e., the same system scenario of Fig. 3a, but based on pure Erbium amplification. System $Q$ decreases and a penalty of $5.4 \mathrm{~dB}$ is measured. It is because EDFA generates more ASE noise than RA, thus more signal power is needed to satisfy the $O S N R_{\text {TARGET }}$ condition. But a higher transmitted power implies a stronger impact of fiber nonlinearities and a consequent stronger impairment on performance. For the pure Erbium, $L_{S P A N}=80 \mathrm{Km}$ SMF scenario, whose results are not graphically reported, penalty increases up to $6.6 \mathrm{~dB}$ and $Q_{M A X}=11.8$ $\mathrm{dB}$, showing that the use of Raman amplification plays a fundamental role in longer spans.

\section{CONCLUSION}

We presented an innovative method that allows the simultaneous optimization of the HFA configuration and dispersion map for multi-span systems. We applied such method to show the difference in using SMF or NZ-DSF as transmission fibers for span lengths of 50 and $80 \mathrm{~km}$. Furthermore, we compared the results of the HFA with EDFA-only: we conclude that HFA systems lead to higher OSNR and are less susceptible to nonlinearities than pure Erbium systems. By increasing the span length from 50 to $80 \mathrm{Km}$ poorer performances were achieved in all studied cases. The use of low dispersion fibers seems to be preferable because it requires a lower amount of DCF.

\section{ACKNOWLEDGMENTS}

The authors would like to thank RSoft Design Group, Inc. for supplying the system simulation tool OptSim ${ }^{\circledR}$. 


\section{REFERENCES}

[1] P. B. Hansen, G. Jacobovitz-Veselka, L. Gruner-Nielsen, A. J. Stentz, "Loss compensation in dispersion compensating fiber modules by Raman amplification," in Proc. Optical Fiber Communication Conference (OFC '98), (San Jose, USA), pp. 20-21, paper Tu.D.1, Feb. 1998.

[2] S. N. Knudsen, "Design and manufacture of dispersion compensating fibers and their performance in systems," in Proc. Optical Fiber Communication Conference (OFC '2002), (Anaheim, USA), paper WU3, pp. 330-332, Mar. 2002.

[3] R. Hainberger, T. Hoshida, T. Terahara, and H. Onaka, "Comparison of span configurations of Raman-amplified dispersion-managed fibers," IEEE Photonics Technology Letters, vol. 14, pp. 471-473, April 2002.

[4] A. Carena, V. Curri, and P. Poggiolini, "On the optimization of hybrid Raman/ErbiumDoped Fiber Amplifiers," IEEE Photonics Technology Letters, vol. 13, pp. 1170-1172, November 2001.

[5] V. Curri, "System advantages of Raman Amplifiers," in Proc. NFOEC 2000, (Denver, USA), vol. 1, paper B1.1, pp 35-46, August 2000.

[6] www.rsoftdesign.com/products/system_simulation/OptSim40

[7] G. P. Agrawal, Nonlinear Fiber Optics, 2nd ed. New York, Academic Press, 2001.

[8] E. Desurvire, Erbium-Doped Fiber Amplifiers, John Wiley \& Sons, 1994.

[9] G. Bosco, A. Carena, V. Curri, R. Gaudino and P. Poggiolini, "Modulation Formats Suitable for Ultra High Spectral Efficient WDM Systems," to be published on IEEE Journal of Selected Topics on Quantum Electronics. 